\title{
Supporting Children with Developmental Disorders: Difficulties and Future Strategies as Perceived by Japanese Public Health Nurses
}

\author{
Chiyori Haga $^{1 *}$, Su Su Maw ${ }^{1}$, Toshiaki Suwa ${ }^{2}$ and Nobuko Ohi ${ }^{3}$ \\ ${ }^{1}$ Community Health Nursing, Graduate School of Health Sciences, Japan \\ ${ }^{2}$ Medical Welfare, Japan
}

${ }^{3}$ Child and Developmental Health Nursing, Graduate School of Health Sciences, Japan

*Corresponding author: Chiyori Haga, Okayama University, 2-5-1 Shikata-cho, Kita-ku, Okayama city, Okayama prefecture, Japan Submission: 毗April 09, 2018; Published: 阱April 13, 2018

\section{Introduction}

In Japan, according to the "Law to Support Persons with Developmental Disabilities" and the project "Healthy Parents and Children 21 (Second Phase)," every prefecture, city, and town needs to identify children affected by developmental disorders and provide support to these children and their parents as early as possible. Public health nurses (PHNs) are responsible for the early detection of cases and complete a diverse range of tasks relating to screening and supporting procedures. The number of children who are waiting to receive specialized medical care in institutions is increasing. Furthermore, the demands for expert personnel such as child psychiatrists, child psychologists, occupational therapists, as well as the establishment of more specialized institutions, are escalating. Various professionals encounter many challenges in identifying children affected by developmental disabilities and in providing effective treatments. Rehabilitative care for children with developmental disorders may be applied to developmental support as for other normal children. It is necessary to promote the skills of professionals relating to maternal and child care. Hence, this study aimed to identify the difficulties experienced by PHNs in providing early care to children with developmental disorders, and to examine ways to improve their performance in the future.

The target population was PHNs from all 27 cities in the Okayama prefecture. Informed consent was obtained from PHNs who were willing to participate in the study conducted from December 2016 to January 2017. Areas of investigation included: developmental status of currently supported children, family structure, and psychological stress experienced by parents. Data were collected using case study forms during short term training sessions involving case studies in the field practice for PHNs. This study was approved by the Ethical Review Committee, Graduate School of Health Sciences (Nursing), Okayama University.
All nine PHN participants were female and the mean age was 36.3 years (yr) with a standard deviation (SD) of 8.8.The mean years of experience as a PHN was $10.6 \mathrm{yr}(\mathrm{SD}=8.2)$, and all were involved in providing health care to children and their parents. The PHNs each reflected upon one case in which they had experienced difficulties in providing supportive care. Of these cases, five were associated with medical examinations, and four of these had involved children who were identified as requiring support at one and half (1.5) years of age.

In Japan, there are two essential health examinations for preschoolers, the first at $1.5 \mathrm{yr}$ of age and the second at $3 \mathrm{yr}$. The finding that most of the participants selected cases relating to the 1.5yr examination may indicate that this examination is particularly crucial in supporting children. According to reports from PHNs, eight out of the nine children selected by participants as each case were boys. The mean age of children at the time of study was $3.2 \mathrm{yr}(\mathrm{SD}=1.2)$ and all were over $2 \mathrm{yr}$ old. Regarding developmental status of the children, the mean birth weight was 2990.6grams $(\mathrm{SD}=188.7$ ) and the mean height was 49.1 centimeters $(\mathrm{SD}=1.7)$. No children had symptoms of premature birth, such as low birth weight, or alater tendency to be overweight or underweight. The skills of the PHNs under consideration were grouped as "methods in supporting caregivers to recognize the developmental problems of their children."

In order to improve nursing skills for PHNs, three strategies were identified. The first relates to the assessment of developmental disability. In order to identify developmental disorders, including autism, PHNs need to use specialized tools such as M-Chat proficiently. It is also essential be able to assess the development of a child through observing and considering family members. The second is acknowledging difficulties during the critical period in 
child development before rehabilitation, and to encourage children to play and develop life skills. Examining the childrearing practices of caregivers could be disadvantageous for children, and so it is necessary to decide how to appropriately to respond to abuse based on each child's developmental stage. It is also important for PHNs to acquire knowledge and skills regarding supporting play and encouraging rehabilitation. Third, and finally, it is necessary to build a relationship with caregivers. Frequent home visits during pregnancy can build a genuine relationship between PHNs and mothers before birth and help encourage preparation for becoming a parent. Furthermore, it is also essential to avoid unwanted pregnancies in adolescents by cooperating with school health providers.

This work was supported by a Grant-in-Aid for Scientific Research (KAKENHI 15K1591307) from the Ministry of Education, Culture, Sports, Science and Technology, Japan.
Creative Commons Attribution 4.0 International License

For possible submissions Click Here

\section{Submit Article}

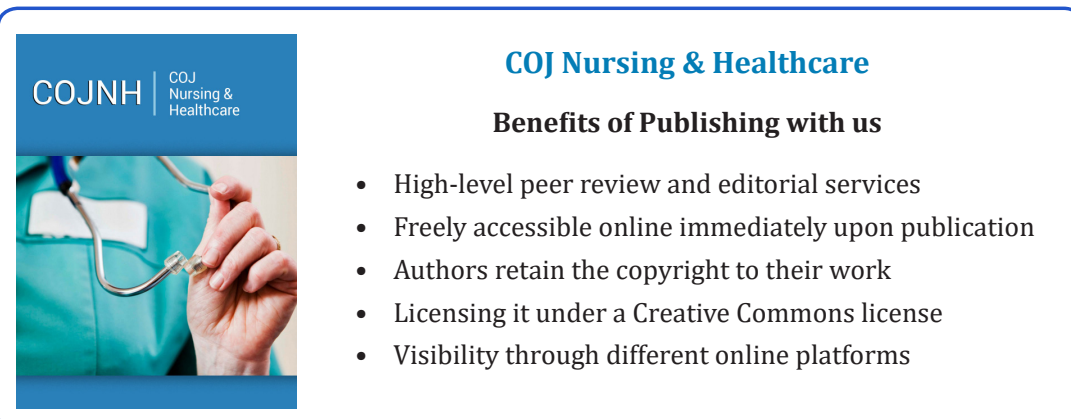

